\title{
噌啉类化合物的合成研究进展
}

\author{
苏琳 侯 卫* \\ (浙江工业大学药学院 杭州 310014)
}

\begin{abstract}
摘要 噌啉类化合物具有抗癌、抗菌、抗病毒、抗炎和镇静作用等多种生物活性. 此外, 噌啉环还是优良的电子受体. 因 此, 噌啉骨架已经成为新材料和新药研发中的优势骨架, 其合成新方法的研究也受到了化学家们的持续关注. 近年来, 碳氢官能团化策略迅猛发展, 大大推动了噌啉类化合物合成新方法的开发. 按照不同的合成策略和反应底物进行分类, 综述了近年来噌啉类化合物合成研究的最新进展.
\end{abstract}

关键词＼cjkstart噌啉; 碳氢官能团化; 偶联; 串联反应

\section{Progress in the Synthesis of Cinnoline Derivatives}

\author{
$\mathrm{Su}, \mathrm{Lin} \quad \mathrm{Hou}, \mathrm{Wei}^{*}$ \\ (College of Pharmaceutical Science, Zhejiang University of Technology, Hangzhou 310014)
}

\begin{abstract}
Cinnolines are "privileged scaffold" in new material and drug research, and development for their extensive biological activities such as anticancer, antibacterial, antiviral, anti-inflammatory, and sedative activities and good electron-accepting ability. As a result, the development of new synthetic routes to this important structure has been actively investigated in recent years. Especially, the $\mathrm{C}-\mathrm{H}$ functionalization strategy has promoted the development of new methods greatly. The recent advances of the synthetic methods to cinnolines based on different synthetic strategies and raw materials are reviewed.
\end{abstract}

Keywords cinnolines; $\mathrm{C}-\mathrm{H}$ functionalization; coupling; tandem reaction

苯并 $[c]$ 哒嗪(benzo $[c]$ pyridazine), 俗称噌啉(cinnoline), 是一类重要的含氮有机杂环化合物(以下统称噌 啉). 噌啉本身具有细胞毒性, 对 Escherichia coli 菌种的 生长有抑制作用 ${ }^{[1]}$, 引入取代基或并环结构的噌啉衍生 物展现出更为广泛的生物活性 ${ }^{[2]}$, 例如抗癌 $(\mathbf{1})^{[3]}$ 、抗菌 $(2)^{[4]}$ 、抗病毒 $(\mathbf{3})^{[5]}$ 、抗炎 $(4)^{[6]}$ 和镇静作用 $(\mathbf{5})^{[7]}$ 等(图 1$)$, 因 此, 它已经成为药物研发中的 “优势骨架”. 此外, 由于 噌啉环是一类良好的电子受体, 具有优良的电子传导能 力, 因此噌啉分子及其配合物在光电材料的开发方面也 有较为广阔的应用空间. 例如化合物 $\mathbf{6}$ 系列是我国科学 家研发的一类新型苂光染料 ${ }^{[8]}$, 化合物 7 被用于 $\mathrm{n}$ 型半 导体的开发 ${ }^{[9]}$, 而苯并 $[c]$ 噌啉类化合物 8 则被广泛应用 于有机发光二极管的研究 ${ }^{[10]}$.

自然界中没有天然的噌啉类化合物存在, 所有文献 中报道的噌啉类化合物都是通过有机合成得到的. 过去
几十年, 由于合成方法有限, 远远不能满足高效合成结 构多样性的噌啉衍生物的需求, 因此, 对于噌啉类化合 物的合成综述报道十分有限 ${ }^{[11]}$, 并且主要集中于对传统 方法的总结. 近十年来, 随着金属催化的偶联反应日渐 成熟以及 $\mathrm{C}-\mathrm{H}$ 活化策略的迅猛发展, 化学家们在传统 方法的基础上应用这些新兴的策略建立了许多合成噌 啉类化合物的新方法, 取得了较好的效果. 对噌啉类化 合物合成的传统方法和近年来发展的新方法进行了归 纳, 并按照不同的合成策略和反应底物进行分类, 综述 了近年来噌啉类化合物合成研究的最新进展(截至 2018 年 7 月). 希望通过这种新的分类归纳思路，从中获得一 些启发，从而设计出更好的合成方法.

\section{1 传统合成方法}

\section{1 噌啉类化合物的经典合成方法}

目前已有五篇论文对噌啉类化合物的经典合成方

\footnotetext{
* Corresponding author. E-mail: houwei@zjut.edu.cn

Received June 15, 2018; revised August 2, 2018; published online September 10, 2018.

Project supported by the Scientific Start-Up Funding from Zhejiang University of Technology (No. 2017116002229) and the China Postdoctoral Science Foundation (No. 2017M612024).

浙江工业大学科研启动基金(No. 2017116002229)及中国博士后科学基金(No. 2017M612024)资助项目.
} 
<smiles>[R]n1c(=O)c2cc(OC)c(OC)cc2c2nnc3cc4c(cc3c21)OCO4</smiles><smiles>O=[N+]([O-])c1ccc2[nH]c3nnc4ccccc4c3c2c1</smiles><smiles>O=C(NCc1ccc(Cl)cc1)c1nnc2ccc(CC3CCOCC3)cc2c1O</smiles>

3

antiviral<smiles>CCN(CC)CCNC(=O)c1nnc2ccc(Cl)cc2c1N</smiles>

sedative activity
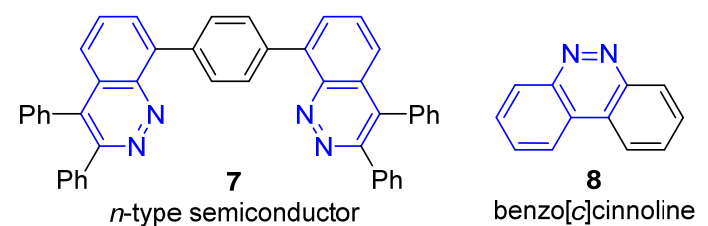

图 1 代表性的噌啉类功能性分子

Figure 1 Representative functional molecules of cinnolines

法进行了综述, 包括 2017 年两篇较新的综述论文. 为避 免赘述的同时又能够保持本文的完整性，我们按照合成 策略对传统合成方法进行归纳总结. 如 Scheme 1 所示, 噌啉类化合物的经典合成方法按照合成策略主要分为 四大类.

Richter 合成法一一邻炔基苯基重氮盐的分子内环 化反应(Scheme 1a). 这种方法是噌啉合成的最早方法, 由 Richter 于 1883 年完成 ${ }^{[12]}$. 其中邻位炔基作为给电子 体进攻分子内的重氮基团，在体系中添加亲核试剂可以 得到不同类型的 3,4-二取代噌啉类化合物 ${ }^{[13]}$. 随后，化 学家们又将分子内的给电子基团拓展至烯基 ${ }^{[14]}$ 和酮类 (烯醇化) $)^{[15]}$. 目前, 这种合成策略已经成为 3,4-二取代 噌啉类化合物最重要的合成方法之一，在药物合成中有 广泛的应用 ${ }^{[16]}$ ，化合物 1 的合成即应用了这种方 法 $^{[3,17]}$. 近年来, 化学家们采用亚硝酸叔丁酯(TBN)合成 重氮盐中间体，可以避免强酸的使用，使反应条件变得 比较温和，在室温下即可顺利进行 ${ }^{[14 e, 18]}$.

Neber-Bossel 合成法一一邻肼基苯乙酸的分子内缩 合反应(Scheme 1b). 这种方法需要在酸性条件下缩合, 因此限制了其广泛使用 ${ }^{[19]}$. 随后，化学家们将亲电基团 更换为炔基 ${ }^{[20]}$ 、羰基 ${ }^{[21]}$ 等官能团，实现了类似转化. 2003 年, Gomaa 小组 ${ }^{[22]}$ 将羧基替换为氰基, 无需酸性条 件，可以得到 3-氨基取代的噌啉化合物.

Barber 合成法一一苯腙化合物的分子内傅克反应 (Scheme 1c). 这种策略由 Barber 小组 ${ }^{[23]}$ 首先发现, 他们 采用 $\mathrm{TiCl}_{4}$ 催化酰氯的分子内傅克反应，实现了 3 -羟基 噌啉类化合物的合成. 在此基础上，化学家们将酰氯基 团替换为醛基 ${ }^{[24]}$ 、氰基 ${ }^{[25]}$ 和酮类 ${ }^{[26]}$ 等亲电官能团，也实 现了类似转化. 这种方法在药物合成中得到了广泛的应

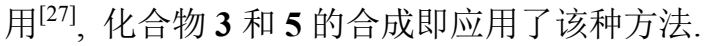

Bräse-Haley 合成法一一邻炔基苯基三氮烯的分子 内环化反应(Scheme 1d). 这种方法本质上是 Richter 合 成法的衍生 ${ }^{[28]}, 1999$ 年, Bräse 小组 ${ }^{[29]}$ 首次将其应用于固 相合成. 该方法的特点在于用氨基保护的重氮盐以稳定 的三氮烯 12 的形式存在, Haley 小组 ${ }^{[30]}$ 通过控制反应条 件可以将其分别转化为噌啉和吲唑化合物, 由于噌啉是 热力学稳定产物, 需要在 $200{ }^{\circ} \mathrm{C}$ 高温下才能得到.
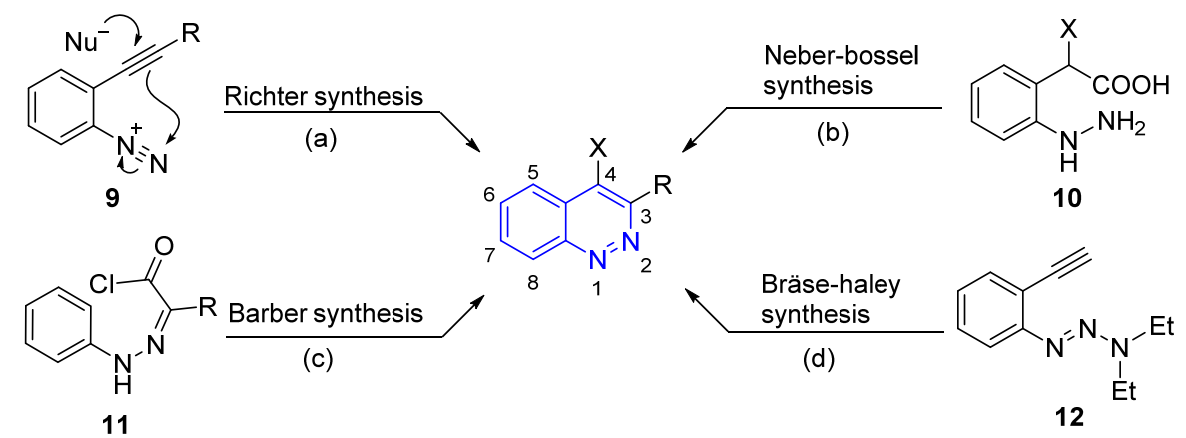

图式 1 噌啉类化合物的经典合成方法

Scheme 1 Classical methods for the synthesis of cinnolines 


\section{2 苯并 $[c]$ 噌啉类化合物的合成方法}

苯并 $[c]$ 噌啉是一类重要的噌啉类化合物, 被广泛 应用于有机光电材料的研究 ${ }^{[10]}$. 由于结构的特殊性, 它 们的合成方法也区别于普通的噌啉类化合物, 因此我们 按照合成策略对其合成方法进行归纳总结. 如 Scheme 2 所示, 苯并 $[c]$ 噌啉类化合物的合成方法主要分为以下 四类.

2,2'二硝基联苯的还原环化反应(Scheme 2a). 采用 $\mathrm{Na}_{2} \mathrm{~S}^{[31 \mathrm{a}]} 、 \mathrm{LiAlH}_{4}{ }^{[31 b]} 、 \mathrm{Sm}^{[32]}$ 或 $\mathrm{Pd} / \mathrm{C}^{[33]}$ 等不同的还原剂 使 13 系列底物发生还原环化反应, 是合成苯并 $[c]$ 噌啉 类化合物最早的方法之一. 尽管这种策略的合成应用较 为广泛 ${ }^{[34]}$, 但在此类反应中难以避免 $N$-氧化物副产物 的生成 ${ }^{[35]}$.

2,2'二二氨基联苯的氧化环化反应(Scheme 2b). Corbett 和 $\mathrm{Holt}^{[36]}$ 在早期报道了以 $\mathrm{NaBO}_{3}$ 为氧化剂的此类反 应, 但反应收率较低; 在此基础上, Caronna 小组 ${ }^{[37]}$ 以 $m$-CPBA 氧化底物后再发生还原反应, 能以较好的收率 得到苯并 $[c]$ 噌啉类衍生物; 2015 年, Minakata 小组 ${ }^{[38]}$ 使 用 $t \mathrm{BuOCl}$ 作为氧化剂, 能够在不产生 $N$-氧化物副产物 的条件下一步合成目标产物. 最近, Cho 小组 ${ }^{[39]}$ 采用 TBN 生成重氮盐的策略, 在室温条件下以中等至较好 的收率得到目标化合物，并且对于酯基、氧基、硝基、 羰基和卤素等基团耐受良好.

偶氮苯的 Schroll 反应或 $[6 \pi]$ 环化反应(Scheme 2c). 1963 年, Went 小组 ${ }^{[40]}$ 采用 $\mathrm{AlCl}_{3}$ 作为催化剂(Schroll 反 应), 在加热条件下得到了一系列噌啉聚环类化合物, 但由于 $E$ 式构型的偶氮不发生反应, 因此收率普遍较 低. 随后, Müller 小组 ${ }^{[4]}$ 采用光照条件发生 $[6 \pi]$ 环化反 应, 使得收率有了明显改善 (光照可以促使 $E$ 式构型的 偶氮转化为 $Z$ 式). 2013 年, Sharma 小组 ${ }^{[42]}$ 同样采用光照 条件, 以 $77 \% \sim 86 \%$ 的收率得到 10 个噌啉并环类化合 物, 这些化合物表现出与对照药吲哚美辛相当的抗炎活 性.
邻芳基苯基重氮盐的分子内傅克反应(Scheme 2d). 这种方法本质上也是 Richter 合成法的衍生，1970 年, Williams 小组 ${ }^{[43]}$ 采用该方法合成了 2-甲氧基苯并 $[c]$ 噌 啉; 2003 年, LaVoie 小组 ${ }^{[44]}$ 利用这种策略合成了一系列 苯并 $[c]$ 噌啉类衍生物, 并以拓扑异构酶 $\mathrm{I}$ 为靶标对这些 化合物的细胞毒性进行了评价；2015 年，杨有军小 组 ${ }^{[8]}$ 应用这一方法合成了一系列具有较大斯托克斯位移 的新型荧光团分子 6 . 这种合成方法具有条件温和、反 应收率高、官能团耐受性好等优点，具有一定的应用空 间 ${ }^{[45]}$.

\section{2 新型合成方法}

以上介绍的经典合成方法在噌啉骨架分子的合成 中占有重要地位，但同时也存在原料制备复杂、需强酸 强碱性反应条件和反应温度高等缺点. 因此, 这些方法 的改进 ${ }^{[14 e, 18]}$ 以及发展原料廉价易得、条件温和、避免强 酸强碱使用的新方法是噌啉类化合物合成研究的发展 方向. 以下我们将近年来开发的新方法按照合成策略分 为四大类: (1)金属催化的偶联反应、(2)炔烃/烯烃的分子 内芳氢化反应、(3) $\mathrm{C}-\mathrm{H}$ 活化策略和(4)其他策略，并逐 一进行介绍.

\section{1 金属催化的偶联反应}

2008 年, Nishida 小组 ${ }^{[46]}$ 采用腙 17 作为底物, 利用 $\mathrm{CuI}$ 催化的分子内 Ullman 反应以定量的收率实现了 3酯基噌啉 18 的合成(Eq. 1). 当采用乙酰基取代腙 19 时, 可以中等收率得到对应的二氢噌啉类化合物 $\mathbf{2 0}$, 并且 通过控制反应条件分别转化为四氢噌啉 21 , 二氢噌啉 22 和 3-酯基噌啉 23 (Scheme 3). 这种方法条件温和, 避 免了强酸强碱的使用，但是原料的制备较为复杂.

2011 年, Yamane 小组 ${ }^{[4]}$ 采用邻碘苯基三氮烯(24)作 为底物, 在零价钯催化下与双取代炔 25 发生串联环化 反应，以中等至较好的收率得到多取代的噌啉类化合物 26 (Eq. 2). 该反应对酯基、氭基、硝基、羰基等基团耐

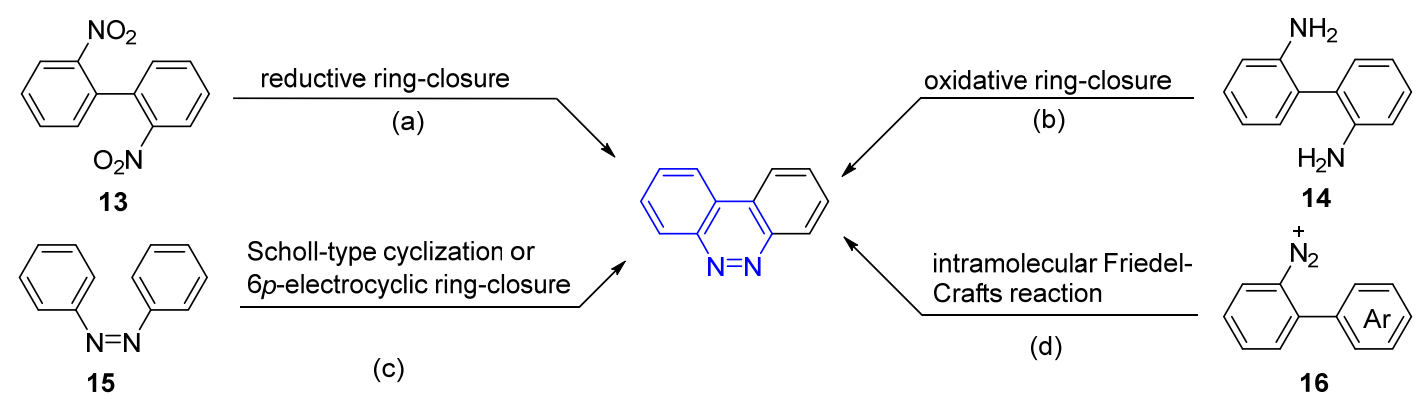

图式 2 苯并噌啉类化合物的经典合成方法

Scheme 2 Classical methods for the synthesis of benzo[c]cinnolines 


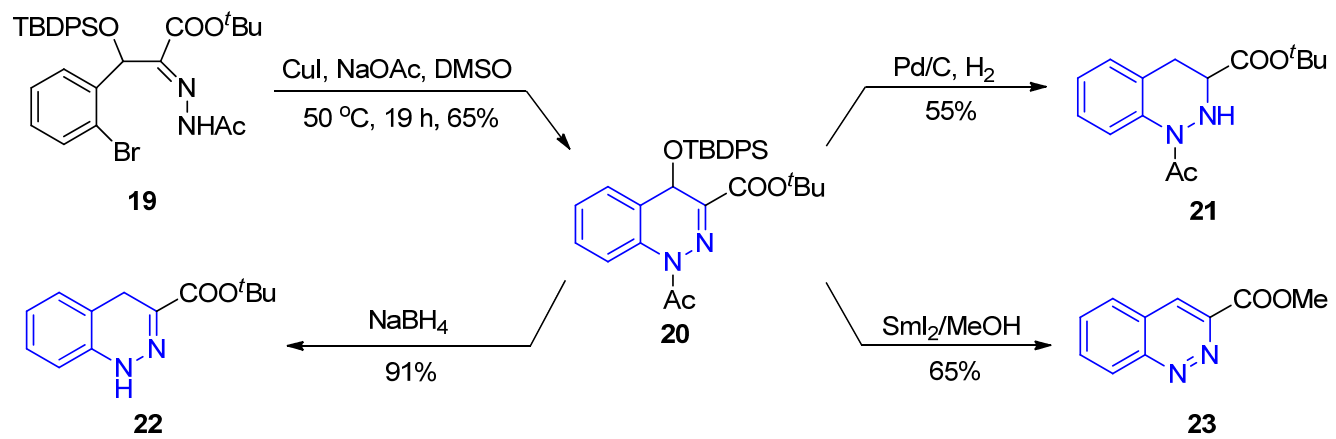

图式 $3 \mathrm{Cu}(\mathrm{I})$ 催化的 $\mathrm{C}-\mathrm{N}$ 键形成

Scheme $3 \mathrm{Cu}(\mathrm{I})$ catalyzed $\mathrm{C}-\mathrm{N}$ bond formation
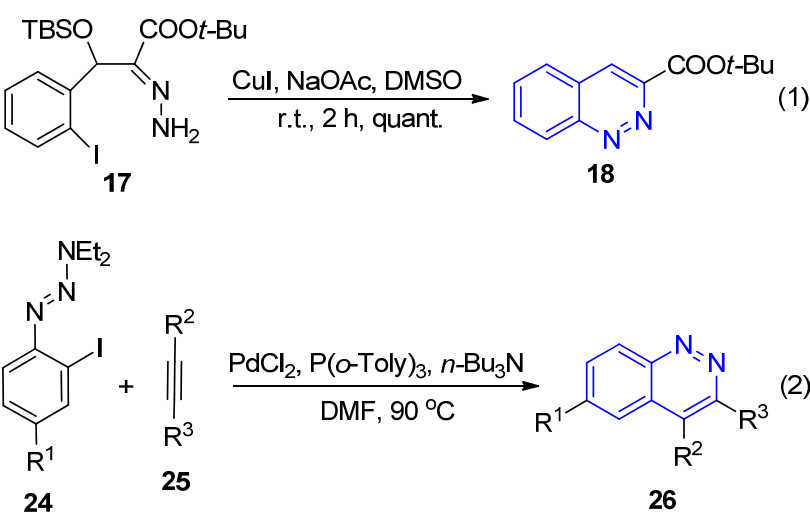

受性良好, 但当采用不对称炔作为原料时，区域选择性 的问题仍有待解决.

2012 年, Willis 小组 ${ }^{[88]}$ 利用 $\mathrm{Cu}(\mathrm{I})$ 催化的串联反应, 采用二卤代物 27 和 1,2-肼二羧酸二乙酯(28)作为原料, 通过两次 $\mathrm{C}-\mathrm{N}$ 键的形成实现了 3-取代二氢噌啉类化合 物 29 的合成, 取得了 $59 \% \sim 91 \%$ 的收率. 通过后续在碱 性乙醇溶液中的脱保护-芳香化步骤, 进一步得到了多 种 3 位官能团化的噌啉类化合物 30 (Scheme 4). 这种分 子间 $\mathrm{C}-\mathrm{N}$ 偶联法与传统方法相比, 原料制备简单, 操 作简便, 为噌啉类化合物的多样性合成提供了新的思 路.

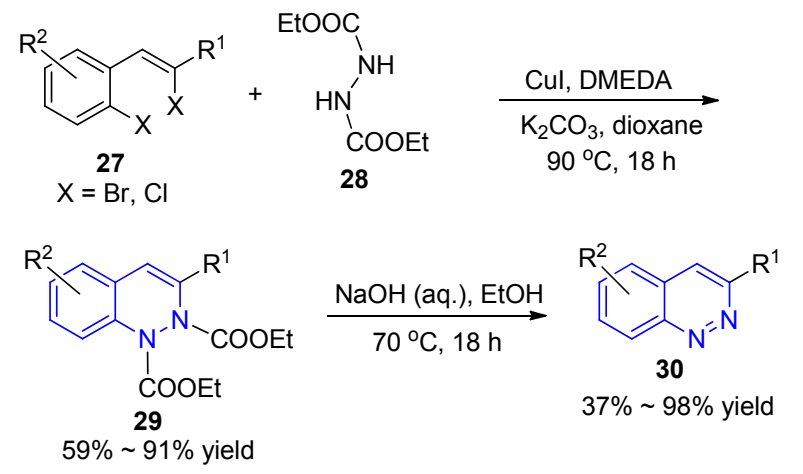

图式 $4 \mathrm{Cu}(\mathrm{I})$ 催化的 $\mathrm{C}-\mathrm{N}$ 偶联法合成噌啉化合物

Scheme 4 Synthesis of cinnolines by $\mathrm{Cu}(\mathrm{I})$ catalyzed tandem $\mathrm{C}-\mathrm{N}$ bond formation
2018 年, Likhar 小组 ${ }^{[49]}$ 以碘代的三氮唑 31 为底物, 经 Sonogashira 偶联和分子内环化的串联反应, 获得了 一系列全新结构的 1,2,3-三唑并噌啉类化合物 32, 收率 $60 \% \sim 85 \%$ (Eq. 3). 该反应具有优异的区域选择性 ${ }^{[50]}$, 选择性地发生 2 位氮原子对炔基的进攻.

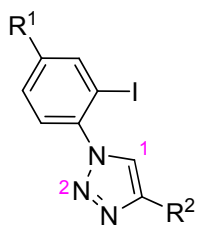

31

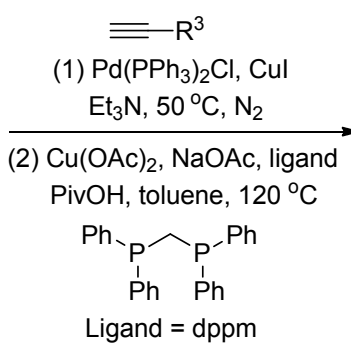

\section{2 炔烃/烯烃的分子内芳氢化反应}

2011 年, Gagosz 小组 ${ }^{[51]}$ 报道了一例 $\mathrm{Au}(\mathrm{I})$ 催化的炔 烃分子内芳氢化反应合成噌啉骨架的新方法. 虽然底物 33 中 2 位的 $\mathrm{N}$ 原子容易与金催化剂发生配位作用，但实 际上并未对反应发生干扰. 该工作首先以 $25 \% \sim 99 \%$ 的 收率完成了对噌啉骨架的构建, 随后在对甲苯磺酸的作 用下发生烯烃移位反应得到产物 35 (Scheme 5). 该方法 采用的底物较易合成，但当 $\mathrm{R}$ 基团为芳基或 $\mathrm{R}^{\prime}$ 基团的取 代位置不在芳环 4 位时，反应存在的区域选择性问题尚 待解决.

2014 年, Gogoi 小组 ${ }^{[52]}$ 采用类似策略, 以烯醛苯腙 36 为底物, 在 $\mathrm{AlCl}_{3}$ 催化下发生分子内的烯烃芳氢化反 应(Eq. 4)得到系列 4-芐基噌啉类化合物 37.

\section{$2.3 \mathrm{C}-\mathrm{H}$ 活化策略}

\subsection{1 腙类底物的分子内氧化脱氢偶联反应}

2012 年, $\mathrm{Ge}$ 小组 ${ }^{[53]}$ 以苯基腙类化合物 $\mathbf{3 8}$ 作为反应 底物，发展了一类铜催化的分子内氧化去氢偶联反应， 经 $\mathrm{sp}^{3}-\mathrm{C}-\mathrm{H}$ 键氧化-环化-芳构化的级联反应过程, 实 现了 3 位芳基取代的噌啉类化合物 $\mathbf{3 9}$ 的直接构建. 这也 是首例通过腙类化合物中 $\mathrm{sp}^{3}-\mathrm{C}-\mathrm{H}$ 键的官能团化实 


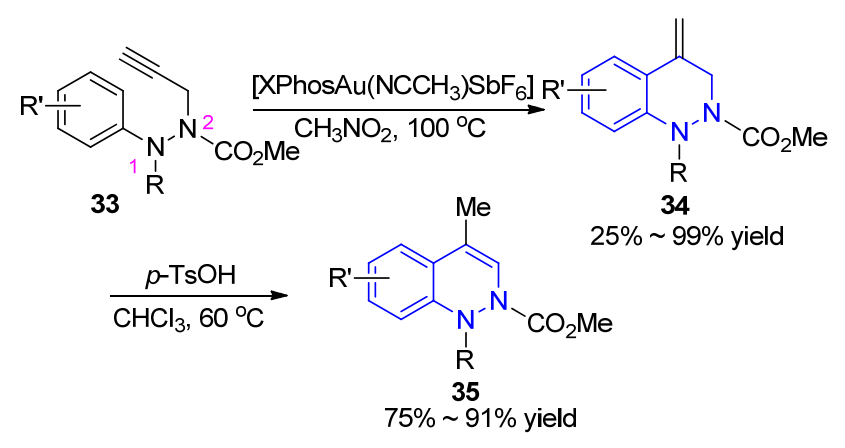

图式 $5 \mathrm{Au}(\mathrm{I})$ 催化的分子内芳氢化反应

Scheme 5 Synthesis of cinnolines by $\mathrm{Au}(\mathrm{I})$ catalyzed intramolecular hydroarylation

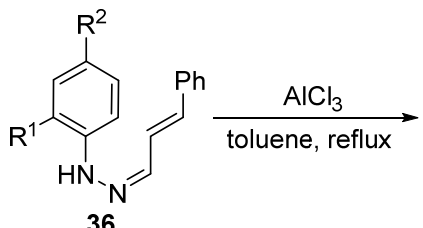

36

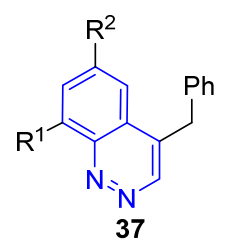

现的铜催化的偶联反应，产率为 $47 \% \sim 96 \%$ (Eq. 5). 该 反应通过自由基机理实现，对卤素、烷基、强吸电子基 $\left(\mathrm{CN} 、 \mathrm{CF}_{3}\right)$ 以及给电子基等都具有很好的适应性, 能够 一步构建噌啉环骨架. 当 $\mathrm{R}^{2}$ 基团处于间位时, 得到具有 一定比例的 5 位和 7 位取代的噌啉混合物. 为避免在 $\mathrm{C}-\mathrm{H}$ 键的氧化过程中产生选择性问题, 设计的底物中 $\mathrm{R}^{1}$ 基团均为芳基，是一种合成 3 位芳基取代的噌啉化合 物的有效方法.<smiles>[R]C/C([R1])=N\N(C)c1ccccc1</smiles>

38

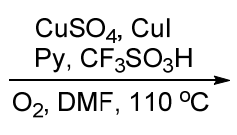<smiles>[R]C1=CC=C2C=C([14CH]=[14CH][14CH]=[14CH]2)N=N1</smiles>

39
2017 年, 许斌小组 ${ }^{[54]}$ 采用醋酸铜水合物作为催化 剂, 以空气作为氧化剂, 通过分子内的氧化去氢偶联芳构化反应实现了 3-取代噌啉类化合物 41 的合成. 这 一方法的原料易于制备, 反应操作简单, 具有潜在的应 用价值，反应收率为 $35 \% \sim 97 \%$ (Eq. 6). 值得一提的是， 当 C-3 位为芳基或烷基取代时均能取得较好的收率，但 C-3 和 C-4 位同时引入取代时收率大幅下降. 有趣的是, 当 $R^{1}$ 基团所在的芳环邻位有 $F 、 O M e 、 \mathrm{NMe}_{2}$ 等官能团 取代时, 即使另一个邻位的 $\mathrm{C}-\mathrm{H}$ 键存在, 反应也能选 择性地发生相对惰性的 $\mathrm{C}-\mathrm{F} 、 \mathrm{C}-\mathrm{O} 、 \mathrm{C}-\mathrm{N}$ 键的断裂.

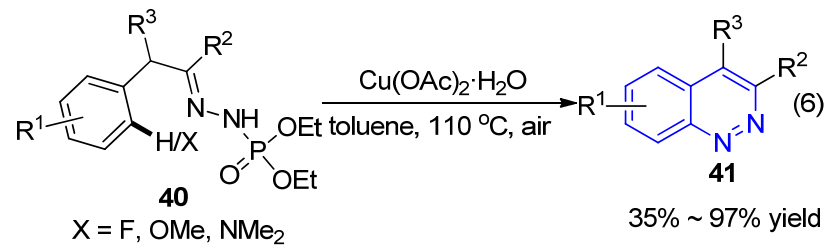

\subsection{2 以碘苯为底物}

2014 年, 张稒羽小组 ${ }^{[55]}$ 以上市药物依达拉奉(42)和碘 苯类化合物 43 作为反应底物, 在钯催化剂和醋酸银的 作用下发生第一次 $\mathrm{C}-\mathrm{H}$ 活化反应，完成了 $\mathrm{C}-\mathrm{C}$ 键的 构建; 在此基础上, 进一步发生钯催化的 $\mathrm{C}-\mathrm{H} / \mathrm{N}-\mathrm{H}$ 氧化去氢偶联反应，完成了 $\mathrm{C}-\mathrm{N}$ 键的构建. 他们采用 这种方法合成了一系列噌啉并环类化合物 44, 收率为 $32 \% \sim 87 \%$ (Eq. 7). 值得一提的是，噌啉并环类化合物 因其结构复杂往往不易合成，由于 1 位和 2 位的芳香氮 原子不能直接进行官能团化，在此之前尚无有效构建 1,2-噌啉并环类化合物的合成方法. 该工作巧妙利用底 物 42 的固有氮原子同时作为导向基团和反应基团，免 去了定位基的引入和脱除引起的步骤浪费. 此反应过程 通过两步一锅法完成, 操作较为操作简便, 是一种合成 此类杂环的有效手段.

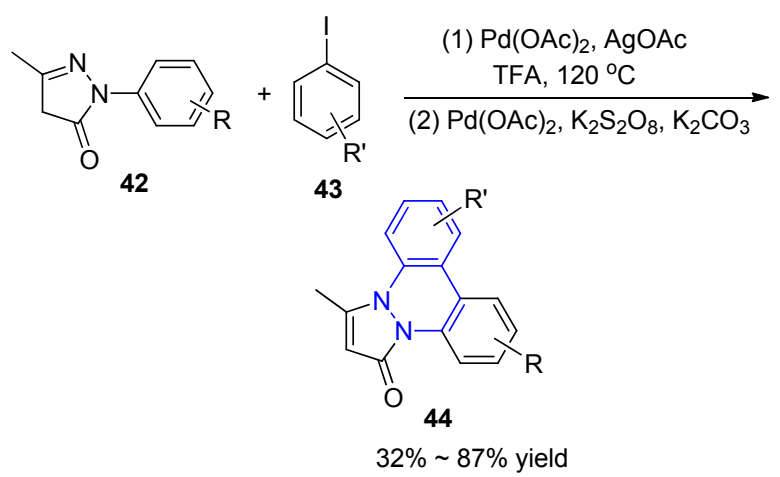

2015 年, Reddy 小组 ${ }^{[56]}$ 采用类似的策略报道了一例 苯并 $[c]$ 噌啉类化合物的合成方法. 他们采用双酯基保 护的苯肼 45 作为底物, 首先在钯催化剂和当量 $\mathrm{AgOAc}$ 的作用下与碘化物 46 发生 $\mathrm{C}-\mathrm{H}$ 活化-芳基化反应形成 $\mathrm{C}-\mathrm{C}$ 键; 随后在过硫酸氢钾和碘苯的作用下发生 $\mathrm{C}-$ $\mathrm{H} / \mathrm{N}-\mathrm{H}$ 氧化脱氢偶联反应形成 $\mathrm{C}-\mathrm{N}$ 键, 同时脱除酯 基形成 48 (Scheme 6). 这一工作的底物适应范围较广, 底物 46 中卤素、羰基、烷基、醚等官能团均可耐受，可 以用于合成多取代苯并 $[c]$ 噌啉类化合物. 但该方法需 要采用当量的银盐作为氧化剂, 并且需要两步才能实 现，降低了反应效率.

\subsection{3 以炔烃为底物}

2013 年, 游劲松小组 ${ }^{[57]}$ 采用偶氮化合物 49 为底物, 以炔烃 50 为偶联试剂，三价铑为催化剂，通过串联反应 成功实现了 3,4-二取代噌啉类化合物的合成. 这一工作 利用与异喹啉合成类似的策略 ${ }^{[58]}$, 将来源广泛、廉价易 得的炔烃应用于噌啉类化合物的构建，通过控制 $\mathrm{R}^{1}$ 基 团的种类和反应条件，可以分别得到噌啉-2-四氟硼酸 盐和噌啉两类产物(Scheme 7)：当 $\mathrm{R}^{1}$ 基团不为叔丁基时, 反应主要得到 2 位噌啉季铵盐 51 , 收率为 $75 \%$ 
<smiles>[R]c1ccc(N(NC(C)=O)C(C)=O)cc1[R16]</smiles>

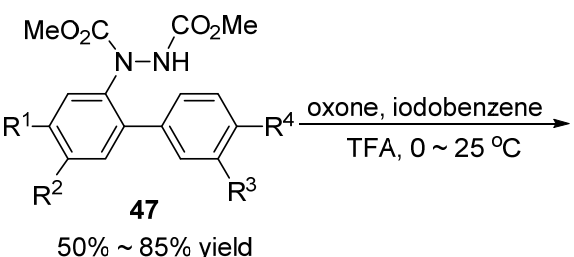

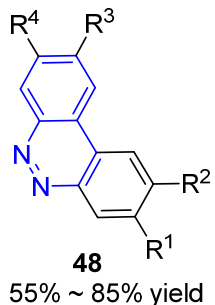

图式 6 通过连续形成 $\mathrm{C}-\mathrm{C}$ 键和 $\mathrm{C}-\mathrm{N}$ 键合成苯并 $[c]$ 噌啉类 化合物

Scheme 6 Synthesis of benzo[c]cinnolines by sequential $\mathrm{C}-\mathrm{C}$ and $\mathrm{C}-\mathrm{N}$ bonds formation

$94 \%$, 在吡啶中进一步加热反应 $8 \mathrm{~h}$ 可以得到噌啉类化 合物 52 (Scheme $7 \mathrm{a}$ ); 当 $\mathrm{R}^{1}$ 基团为叔丁基时, 对于烷基 取代的炔烃, 通过调整反应条件, 可以直接得到电中性 的噌啉化合物 53 (Scheme 7b). 该反应对酯基、氭基、 卤素和酮等官能团均可耐受, 对于不对称的炔烃具有较 好的区域选择性，同时易发生副反应的端炔也能顺利发 生反应，是一种构建 3,4-二取代噌啉的有效方法.

无独有偶, Cheng 小组 ${ }^{[59 a]}$ 几乎在同时发表了类似的 工作. 他们同样以偶氮化合物作为底物, 以炔烃作为偶 联试剂, 三价铑作为催化剂, 不同的是体系中采用六水 合四氟硼酸铜作为氧化剂, 以叔丁醇为反应溶剂, 在 $70{ }^{\circ} \mathrm{C}$ 条件下发生反应, 得到 3,4-二取代的 2 位噌啉季铵
盐 56 (Eq. 8), 收率水平与游劲松小组相当, 产物可以进 一步转化为吲哚和吲哚并吲哚两类化合物. 他们在机理 研究过程中成功合成并分离得到了环化铑中间体 $\mathbf{5 8}$, 将其用于催化反应，以 $94 \%$ 的收率顺利得到了目标产 物，进而验证了反应可能经历的碳氢活化一环化过程. 作者据此提出了可能的反应机理(Scheme 8), 底物 57 首 先与金属铑物种反应生成环化铑中间体 $\mathbf{5 8 , 5 8}$ 与炔烃络 合形成中间体 59, 发生顺式插炔反应后进一步生成七 元环中间体 60 , 随后发生还原消除反应得到噌啉季铵 盐 61 .

在此基础上, 2016 年, Cheng 小组 ${ }^{[59 b]}$ 利用相同的策 略, 采用 $\mathrm{Co}(\mathrm{III})$ 催化剂，以 $78 \% \sim 90 \%$ 的收率得到了 3,4-二取代的噌啉季铵盐 64 (Eq. 9). 值得一提的是, 催 化剂的种类在这个反应中十分重要. 在篮选过程中, 除 了 $\left[\mathrm{CoCp} *(\mathrm{CO}) \mathrm{I}_{2}\right]$ 之外, 其它的二价钴和三价钴催化剂 均无效. 作者选取反应得到的代表性噌啉类化合物用于 光学性质的研究, 发现部分化合物在 $490 \mathrm{~nm}$ 波长位置 展现出强的蓝色菼光发射，说明这些化合物在蓝色苂光 有机发光二极管方面具有潜在的应用价值.

2014 年, 张期小组 ${ }^{[60]}$ 利用类似的策略以廉价易得 的依达拉奉 $(65)$ 和炔烃 66 作为原料, 三价铑作为催化 剂，六氟异丙醇为溶剂，在 $70{ }^{\circ} \mathrm{C}$ 条件下反应，以中等 到优秀( $41 \% \sim 96 \%)$ 的收率得到了系列吡唑啉酮并噌啉 类化合物 67 (Eq. 10), 硝基、酯基等敏感基团均能良好 耐受. 另外, 由于依达拉奉是一种已知的上市药物, 这 一方法也为依达拉奉的修饰提供了新的思路.

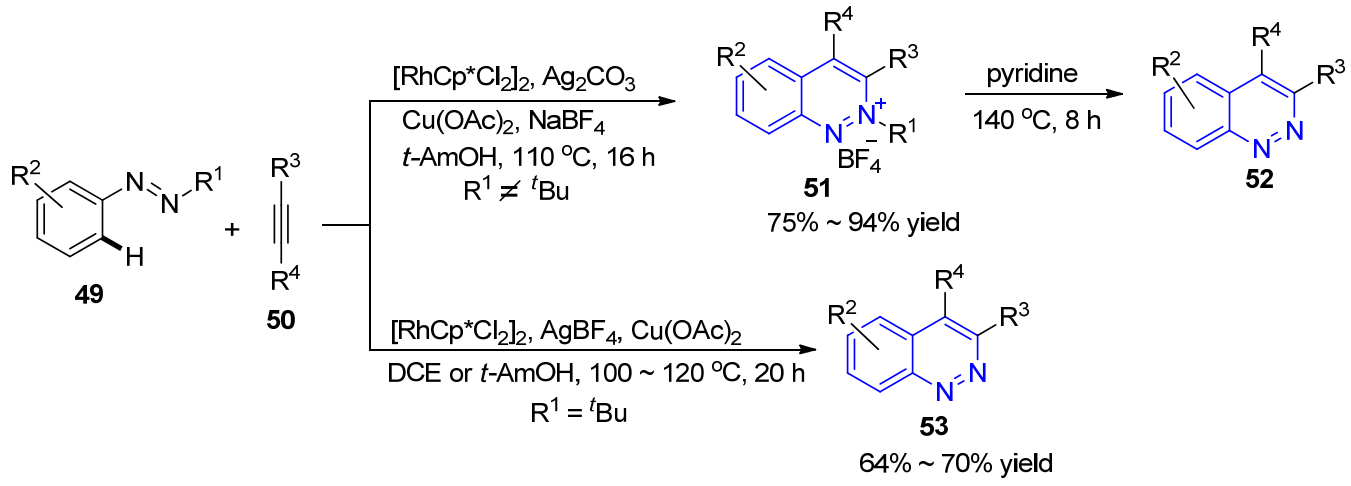

(a)

图式 $7 \mathrm{C}-\mathrm{H}$ 活化/环化反应合成噌啉类化合物

Scheme 7 Synthesis of cinnolines by $\mathrm{C}-\mathrm{H}$ activation/annulation<smiles>[R]C#[R]C#[R]</smiles>

$\frac{\left[\mathrm{RhCp}{ }^{*} \mathrm{Cl}_{2}\right]_{2}, \mathrm{Cu}\left(\mathrm{BF}_{4}\right)_{2} \cdot 6 \mathrm{H}_{2} \mathrm{O}}{t-\mathrm{BuOH}, 70^{\circ} \mathrm{C} \text { air } 16 \mathrm{~h}}$

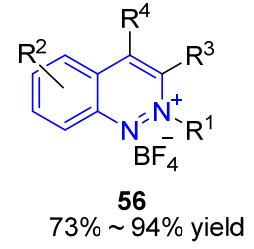

Chin. J. Org. Chem. 2019, 39, 363 376 


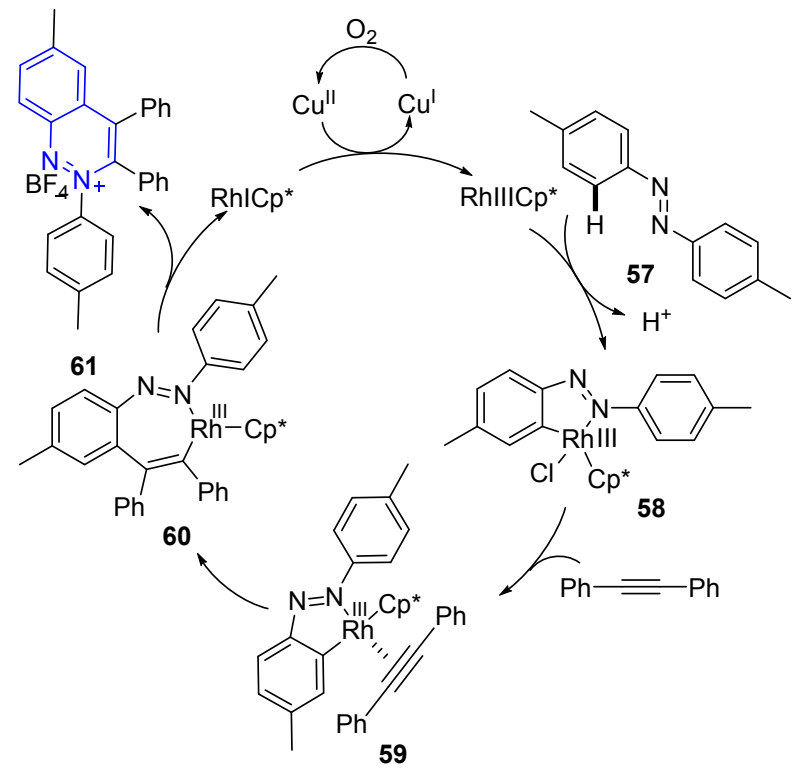

图式 8 噌啉季铵盐合成的反应机理

Scheme 8 Proposed mechanism for cinnolinium-salt formation<smiles>[R]C#C[CH][R]</smiles>

62

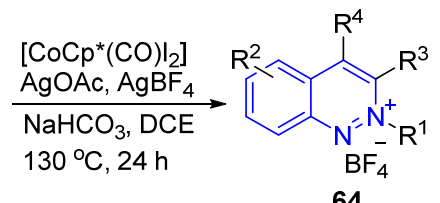

$78 \% \sim 90 \%$ yield

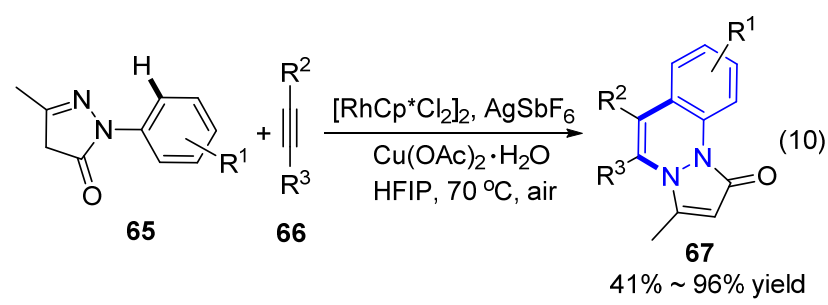

2016 年, Gandhi 小组 ${ }^{[61]}$ 以相对廉价的二价钉作为金 属催化剂, 报道了另一例噌啉并环类化合物的合成方 法. 该方法采用哒嗪酮和酞嗪酮 68 作为底物, 与苯基炔 丙醇 69 发生区域选择性的氧化环化反应，同样以分子 内固有官能团作为定位基, 醋酸作为仅有的副产物脱 除, 展示出较好的原子经济性. 该反应底物适应性广
泛，对酮羰基、硝基等官能团耐受性良好，产率为 $65 \%$ ～94\% (Eq. 11). 值得一提的是，羟基通常被视为一 种离去能力较差的基团, 然而在这个反应中得到的却是 炔丙醇底物去氧化的产物, 其反应机理值得进一步深入 研究.

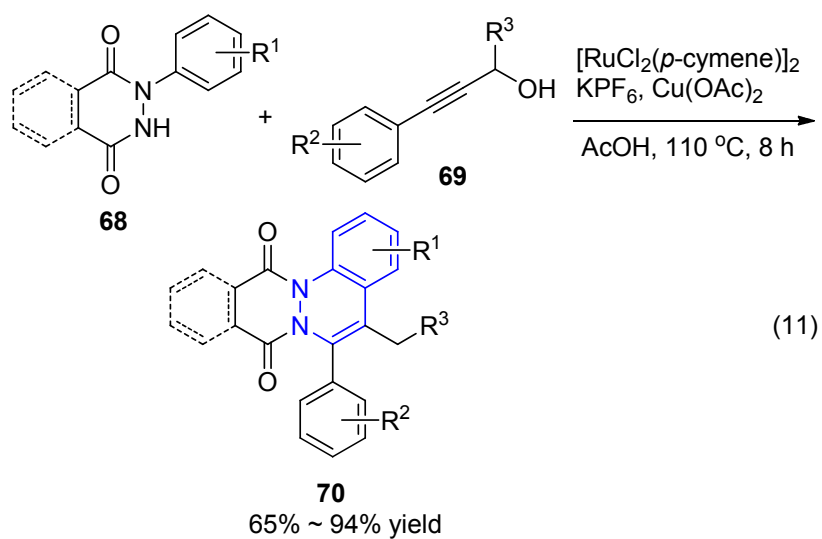

2016 年, Perumal 小组 ${ }^{[62]}$ 采用与 Gandhi 小组报道的 工作中类似的底物 71, 在三价铑的催化作用下合成了 哒嗪并噌啉类化合物 72 (Eq. 12), 收率与上述工作维持 在同一水平. 除此之外, 他们还以吲唑衍生物 73 作为反 应底物与不同的炔发生反应，合成了吲唑酮并噌啉类化 合物 74 (Eq. 13). 这些杂环化合物展现了良好的聚集诱 导发光性质，同时，良好的生物相容性使得它们具有用 于细胞成像研究的潜在价值.

\subsection{4 以重氮化合物作为底物}

2015 年，易伟小组 ${ }^{[63 a]}$ 报道了以重氮化的麦氏酸 76 作为偶联试剂, 与依达拉奉衍生物 75 发生三价铑催化 的 $\mathrm{C}-\mathrm{H}$ 活化/环化反应，合成了 9 个噌啉酮并环类衍生 物 77, 产率为 $43 \% \sim 90 \%$ (Eq. 14). 该反应底物便于合 成, 不需添加其他配体, 体系简单. 2016 年, 他们 ${ }^{[63 b]}$ 采 用三价铱为催化剂, 甲醇作为溶剂实现了类似转化, 反 应时间缩短至 $0.5 \mathrm{~h}$ (Eq. 15).

同年, Lee 课题组 ${ }^{[64]}$ 同样以 76 作为偶联试剂，与芳 基偶氮底物 80 在三价铑的催化作用下发生 $\mathrm{C}-\mathrm{H}$ 活化/ 环化反应，实现了 2-芳基取代噌啉酮 81 的构建. 在

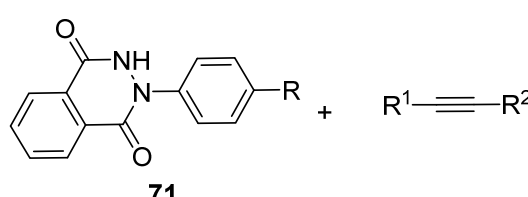<smiles>O=c1[nH]n(-c2ccccc2)c2ccccc12</smiles>

$\frac{\left[\mathrm{RhCp}^{*} \mathrm{Cl}_{2}\right]_{2}, \mathrm{AgSbF}_{6}}{\mathrm{Cu}(\mathrm{OAc})_{2} \mathrm{H}_{2} \mathrm{O}, \mathrm{t}-\mathrm{AmOH}, 6 \mathrm{~h}}$ $100^{\circ} \mathrm{C}$

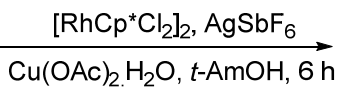
$110^{\circ} \mathrm{C}$

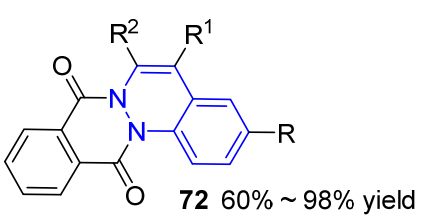<smiles>[R]c1c([R])n2c(=O)c3ccccc3n2c2ccccc12</smiles>

$\mathbf{7 4} 75 \% \sim 94 \%$ yield 


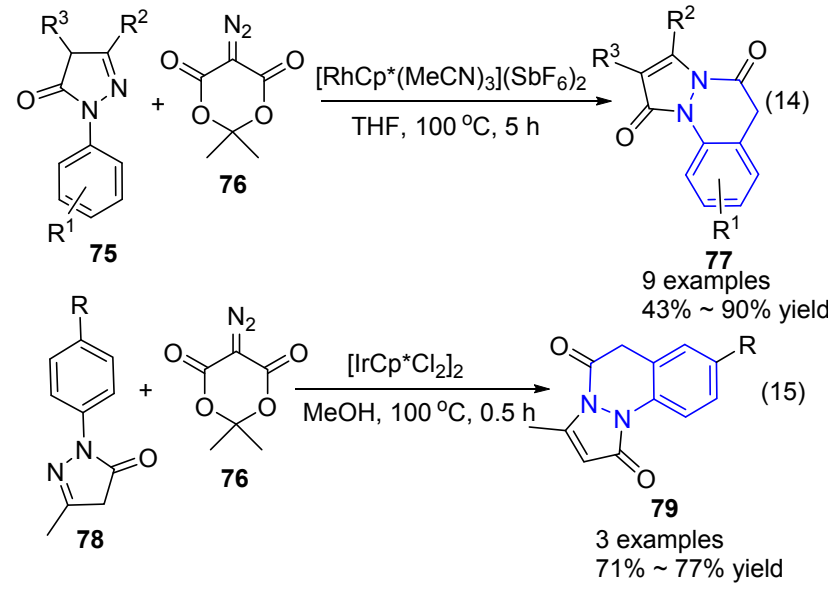

$\left[\mathrm{RhCp}^{*} \mathrm{Cl}_{2}\right]_{2} / \mathrm{AgSbF}_{6}$ 的催化体系下, 以乙醇为溶剂, 在 $80{ }^{\circ} \mathrm{C}$ 条件下发生反应，取得了 $45 \% \sim 99 \%$ 的收率(Eq. 16). 反应产物中 C-4 位的酯基为进一步官能团转化提 供了有利条件. 在这个反应中, 当底物为非对称的偶氮 化合物时, 不能得到选择性的产物, 而是以一定比例得 到两种混合产物. 反应机理如 Scheme 9 所示, 通过亲电 去质子化得到环化铑中间体 83 后, 底物 76 与其发生络 合作用进而形成中间体 $84 ; 84$ 可能在形成铑卡宾中间体 85 后发生迁移插入反应得到中间体 86, 也可能直接发 生 1,2-芳基迁移反应得到中间体 86 , 随后发生质子化作 用形成 87, 进一步得到产物 88.

几乎同时, Kim 小组 ${ }^{[65]}$ 报道了类似的反应. 他们采 用相同的催化体系，以甲醇作为溶剂，得到相应的甲酯 化产物(Eq. 17), 但是收率较低, 并且底物大都局限在对 称的芳基偶氮化合物. 有趣的是, 在与重氮化的丙二酸 二甲酯(91)反应时, 增加重氮的用量至 3 equiv., 可以得<smiles></smiles>

到噌啉酮化合物 C-8 位进一步 $\mathrm{C}-\mathrm{H}$ 烷基化的产物 92 (Eq. 18)

2016 年，林爱俊和姚和权小组 ${ }^{[66]}$ 报道了在 $\mathrm{Rh}(\mathrm{III})$ 的催化作用下，偶氮底物 93 和重氮化底物 94 发生 C$\mathrm{H}$ 烷基化/环化反应，一步合成了 3,4-二取代的噌啉类化 合物 95 (Eq. 19). 该反应在 $50{ }^{\circ} \mathrm{C}$ 下即可发生, 条件温 和，反应体系简单，底物适用范围广，除了硝基取代的 底物收率较低外, 其他常见的取代基都能获得很好的产 率. 另外, 由于底物中的 Boc 基团在反应完成后自动脱 除，避免了不对称二芳基偶氮底物引起的区域选择性问 题，这些优点使得该方法成为一种有效地合成 3,4-二取 代噌啉化合物的方法.

同年, 朱进小组 ${ }^{[67]}$ 采用 1-取代苯肼 96 作为底物，重 氮化合物 97 作为偶联试剂, 三价铑为催化剂, 通过串联 反应实现了 1,3,4-三取代噌啉骨架化合物 98 的合成. 该 反应以甲醇为溶剂，以醋酸或碱金属醋酸盐作为唯一添 加剂，反应体系简单(Eq. 20). 反应在室温下即可进行, 对氧基、酯基等敏感基团均能良好耐受，能够以良好至

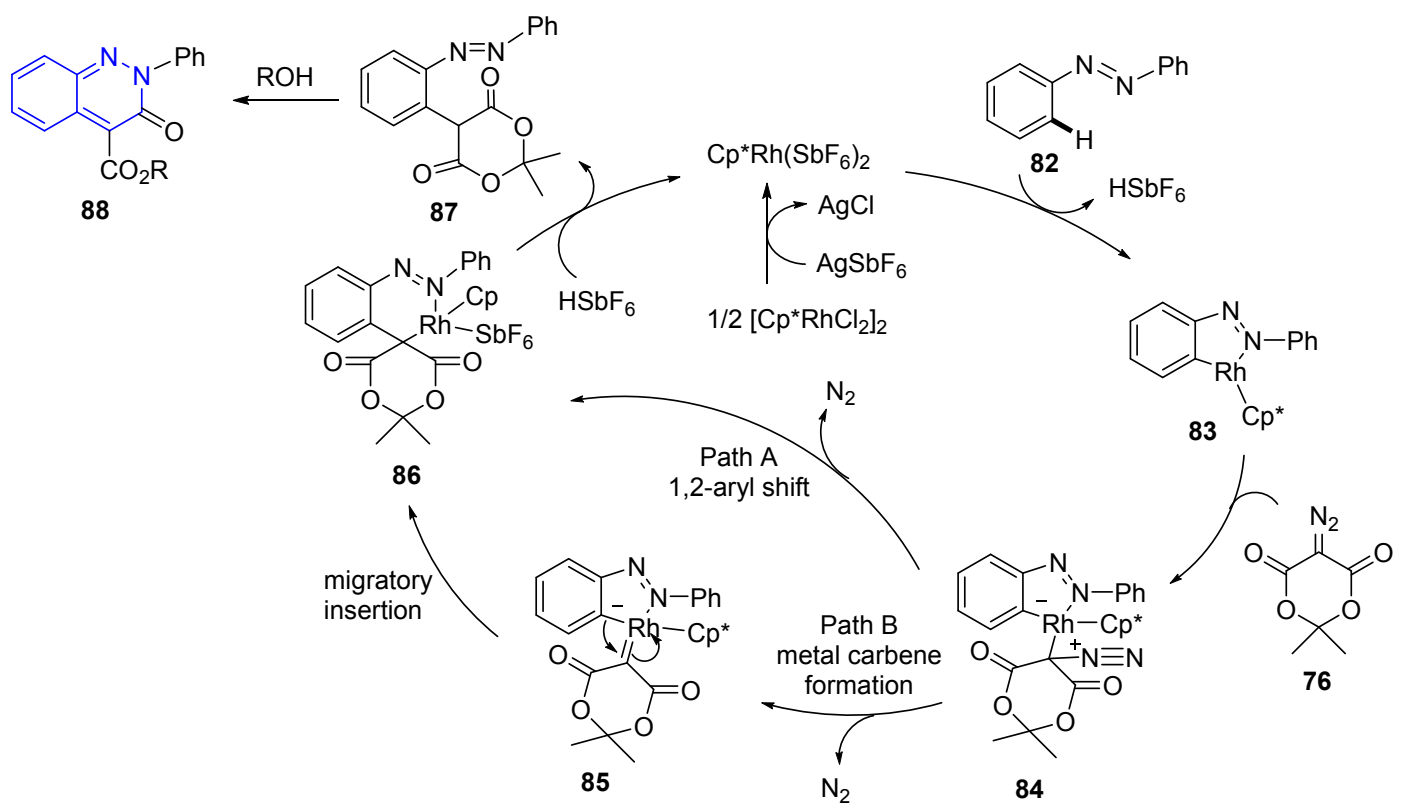

图式 9 与重氮化合物的 $\mathrm{C}-\mathrm{H}$ 活化/环化过程机理

Scheme 9 Proposed mechanism of $\mathrm{C}-\mathrm{H}$ activation/annulation with diazo compounds 
<smiles>[R][R]c1cccc(N=Nc2cccc(N=Nc3cc[R]([H])cc3)c2)c1</smiles>

$30 \% \sim 77 \%$ yield<smiles>[R1]c1cccc(N=Nc2cccc([R])c2)c1</smiles>

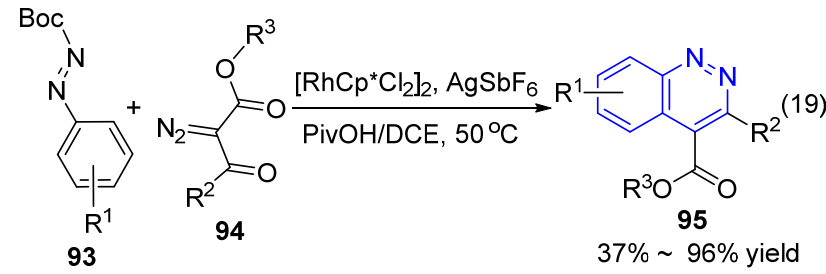

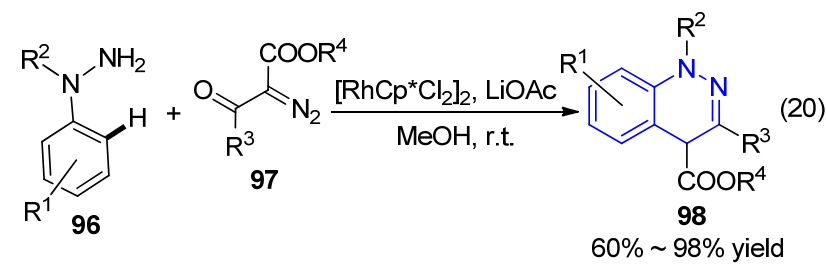

优秀的收率(60\% 98\%)获得目标产物，具有较好的应 用价值.

2018 年, 林爱俊和姚和权小组 ${ }^{[68]}$ 采用菲尼酮 99 为 底物, 以 $\alpha$-羰基重氮类化合物 100 为偶联试剂, 发展了 一类三价铑催化的 $\mathrm{C}-\mathrm{H}$ 活化/环化反应, 合成了一系列 二氢吡唑酮并 $\left[1,2-a\right.$ ]噌啉类化合物 101. 该反应在 $40{ }^{\circ} \mathrm{C}$ 下发生，条件温和，对硝基、氰基、酮羰基和端位烯基 等敏感基团均能良好耐受，收率为 44\%～87\% (Eq. 21). 在这个工作中, 反应仅生成无毒无害的 $\mathrm{N}_{2}$ 和 $\mathrm{H}_{2} \mathrm{O}$ 副产 物, 具有较好的原子经济性, 符合现代绿色化学的发展 方向.

2018 年, 李兴伟小组 ${ }^{[69]}$ 采用偶氮 102 为底物, 在三 价铑催化下, 与 $\alpha$-羰基重氮类化合物 103 发生 $\mathrm{C}-\mathrm{H}$ 活 化/环化反应, 建立了合成噌啉三氟甲磺酸盐 104 的新方 法(Eq. 22). 该反应在室温进行, 反应体系简单, 但当采 用不对称偶氮底物时区域选择性的问题仍待解决.

\subsection{5 以 $\alpha$-拟卤代酮为底物}

2017 年, 黄湧小组 ${ }^{[70]}$ 以菲尼酮 105 作为底物, $\alpha$-甲 磺酰氧基酮 106 作为偶联试剂, 报道了一例 Rh(III)催化 的 $\mathrm{C}-\mathrm{H}$ 活化/环化反应合成噌啉并环骨架. 在这个工

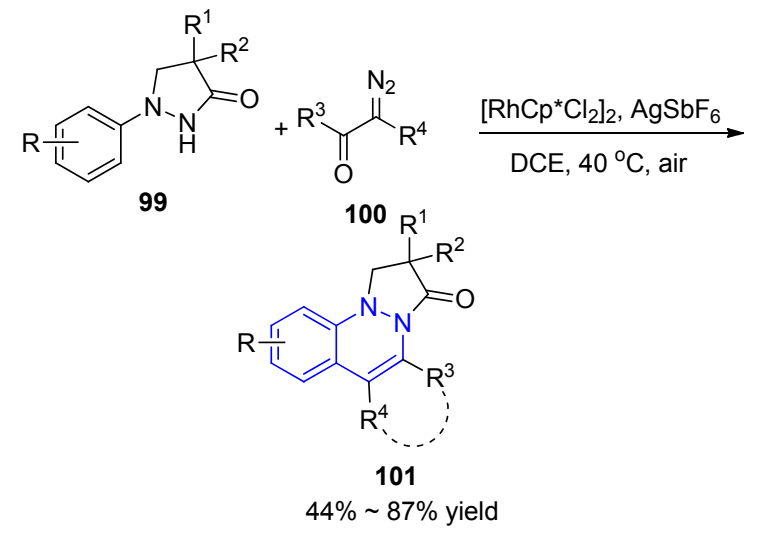

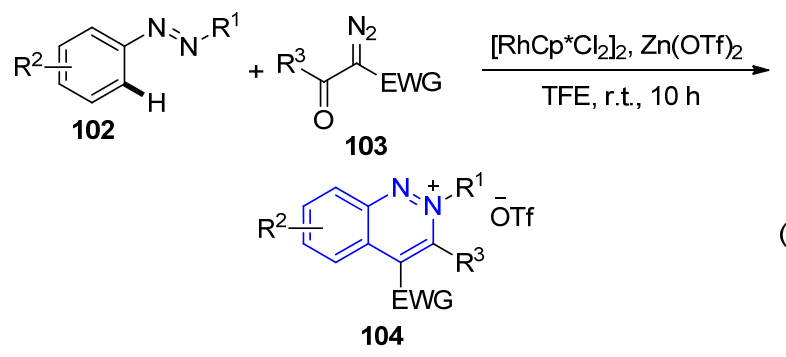

作中，碱的种类可以调节后续的脱水步骤是否发生，从 而控制反应的产物类型. 当采用 $\mathrm{NaOCN}$ 作为碱时, 反 应停留在醇的阶段(Eq. 23); 当采用 $\mathrm{NaOPiv}$ 作为碱时, 反应进一步脱水(Eq. 24). 该反应条件温和, 底物适应性 好，卤素、硝基、三氟甲基、三氟甲氧基等取代的底物 都能获得很好的收率, 且反应能够放大至克级进行.

\section{4 其他合成策略}

\subsection{1 邻三氟甲基苯腙的 $[6 \pi]$ 环化反应}

1995 年, Kiselyov 小组 ${ }^{[71 \mathrm{a}]}$ 发展了一类 4-氨基噌啉类 化合物的新型合成方法. 1999 年, 他们 ${ }^{[71 b]}$ 利用该方法以 邻三氟甲基苯肼与芳香醛缩合成苯腙类底物 109 后，在 强碱 NaHMDS 的作用下得到一系列 3-芳基-4-氨基噌啉 类化合物 116. 三氟甲基是该反应的必需基团，反应机 


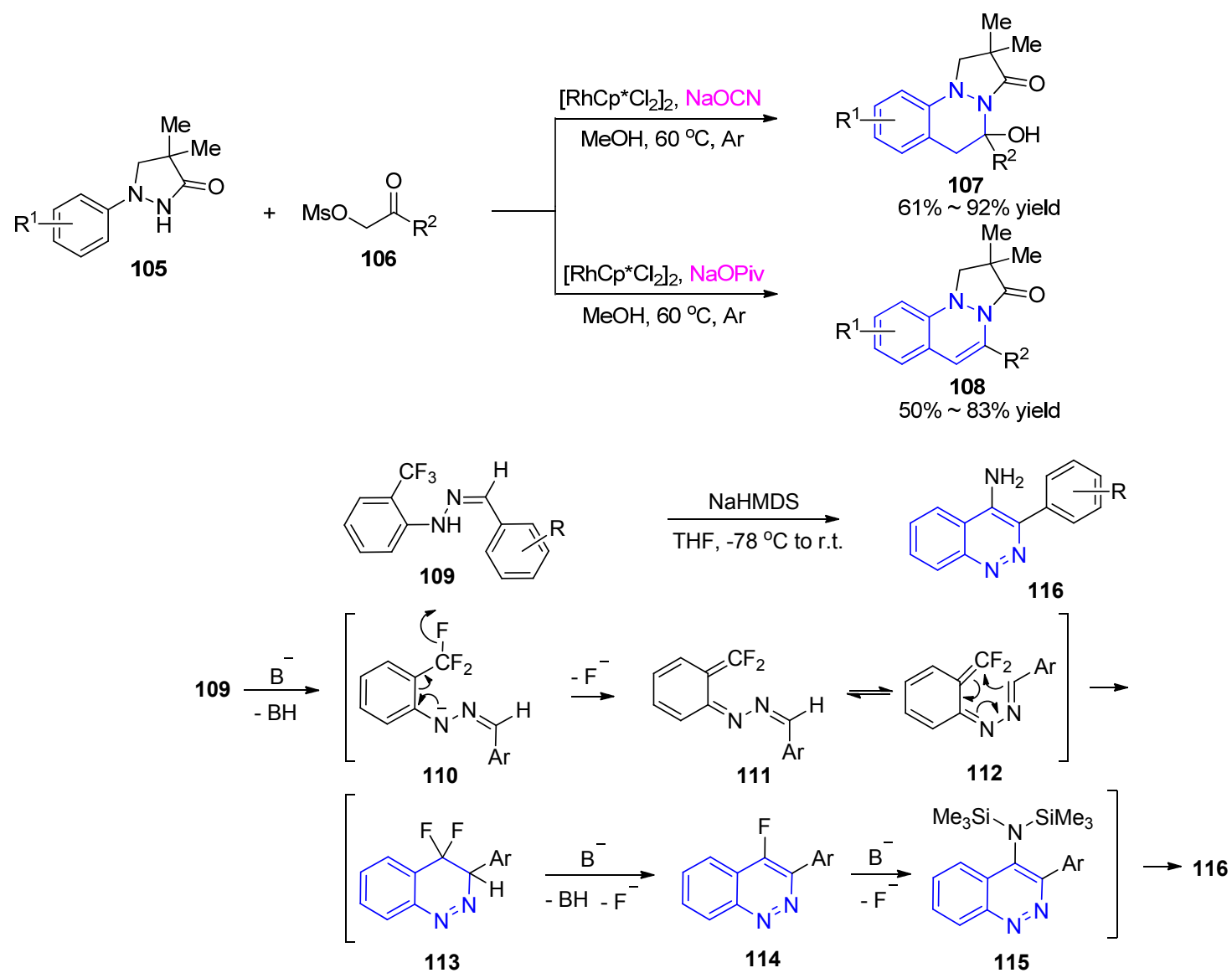

图式 10 邻三氟甲基苯腙的 $[6 \pi]$ 环化反应机理

Scheme $10[6 \pi]$ annulation of $o$-trifluoromethyl phenylhydrazones and proposed mechanism

理如 Scheme 10 所示. 首先在碱的作用下拔氢形成氮负 离子 110 , 为稳定负电荷发生分子内电荷转移同时离去 氟离子得到中间体 111 , 进一步发生 $[6 \pi]$ 环化反应得到 中间体 113, 随后在碱的作用下芳构化得到中间体 114, 再与碱发生芳香亲核取代得到中间体 115 , 酸处理后即 得到目标化合物 116.

\subsubsection{PTAD 的 D-A 反应}

4-苯基-3H-1,2,4-三唑啉-3,5-二酮(PTAD)是一种高 活性的亲双烯体, 在有机合成中常常用作氮气的合成等 价物参与反应 ${ }^{[72]} .2009$ 年, Alajarin 小组 ${ }^{[73]}$ 采用烯酮亚胺 117 作为底物, 与 PTAD 发生 D-A/ene 串联反应得到中 间体 118, 最后在 $\mathrm{KOH}$ 的作用下发生消除反应得到 3氨基取代噌啉化合物 119 (Scheme 11).

\subsection{3 邻硝基苯乙腈的环化反应}

2009 年, 杨春皓小组 ${ }^{[74]}$ 采用邻硝基苯乙腈 $\mathbf{1 2 0}$ 作为 底物, 利用苯基格氏试剂对氰基进攻引发的串联环化反 应实现了系列二氢噌啉类化合物 121 的合成, 部分化合 物对急性早幼粒白血病细胞 HL-60 表现出中等抑制活 性(Eq. 25).<smiles>[R]C(=C=N[Al])c1ccc([R7])cc1</smiles><smiles>[R]c1ccc2c([R])c(N[Al])nnc2c1</smiles>

图式 11 烯酮亚胺与 PTAD 的反应 Scheme 11 Reaction of ketenimines with PTAD

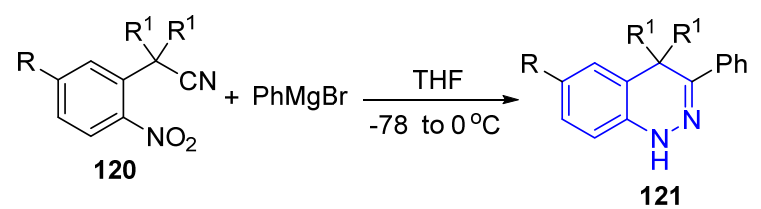

2.4.4 分子内芳香亲核取代反应

2013 年, 宫平小组 ${ }^{[75]}$ 以苯腙 122 作为原料, 在碱性 
条件下发生分子内的芳香亲核取代反应，得到系列 4-氧 代噌啉-3-羧酸化合物 123, 进一步合成得到了系列小分 子 c-Met 抑制剂 124, 优选化合物的 $\mathrm{IC}_{50}$ 值为 0.59 $\mathrm{nmol} / \mathrm{L}$, 展示出优异的抗肿瘤活性(Scheme 12).<smiles>[R]OC(=O)C(Nc1ccccc1F)C(C(=O)OCC)C(=O)c1ccc([R])cc1F</smiles><smiles>[R]c1ccc2c(=O)c(C(=O)O)nn(-c3ccccc3)c2c1</smiles><smiles>[R]CCCOc1cc2nccc(Oc3ccc(NC(=O)c4nn(-c5ccccc5)c5cc(PC#CC)ccc4c5=O)cc3F)c2cc1OC</smiles>

图式 12 苯腙的分子内芳香亲核取代反应 Scheme 12 Intramolecular $\mathrm{S}_{\mathrm{N}} \mathrm{Ar}$ reaction of phenylhydrazones

\section{4 .5 傅克一氧化脱氢偶联反应}

2016 年, Sabitha 课题组 ${ }^{[76]}$ 采用咪唑并环类化合物 125 作为底物, 首先与偶氮二甲酸二异丙酯(DIAD)发生 C-3 区域选择性的联氨化反应, 再在 PIDA 的作用下发 生 $\mathrm{C}-\mathrm{H} / \mathrm{N}-\mathrm{H}$ 氧化去氢偶联反应, 完成了两个 $\mathrm{C}-\mathrm{N}$ 键 的构建, 合成了一系列噌啉并杂环类化合物 $\mathbf{1 2 8}$ (Scheme 13). 通过控制反应条件, 可以分离得到联氨化 中间体 118, 随后通过控制 PIDA 的当量, 分离得到了偶 氮中间体 127, 127 可以继续在 PIDA 的作用下发生环合 得到目标产物 128. 该方法实际上是通过两步反应来实 现目标噌啉环的合成, 但可以经一锅法完成. 反应过程 中无需过渡金属参与, 条件温和. 基于该方法, 作者合
成了三种杂环并噌啉类化合物 129 131. 2017 年，汤日 元小组 ${ }^{[77]}$ 也用类似的策略合成了多种杂环化合物，其 中报道了三个构建噌啉并环类化合物的例子.

\subsection{6 基于苯炔中间体的环化反应}

2015 年, Chenoweth 小组 ${ }^{[78]}$ 以四嗪 $\mathbf{1 3 3}$ 为底物, 与 原位生成的苯炔中间体发生 Diels-Alder 反应，得到了系 列噌啉类聚环化合物 134 (Eq. 26). 134 具有较好的荧光 性质，其光学性质可通过取代基调控，具有应用于肝细 胞影像检测的潜力. 该反应采用一锅法在室温条件下进 行，对氧气不敏感，反应完成时间不到 $5 \mathrm{~min}$, 较为便 捷, 但收率较低.

2016 年, $\mathrm{Wu}$ 小组 ${ }^{[79]}$ 报道了一例通过苯炔中间体、 偶氮中间体与 $\alpha$-澳代酮底物发生的三组分环化反应合 成 3-取代噌啉类化合物的方法, 产率 35\% 70\%. 他们 提出的可能反应机理如 Scheme 14 所示: 化合物 $\mathbf{1 3 5}$ 在 氟化铯的作用下原位生成苯炔中间体 138; 与此同时, 对甲苯磺酰肼(136)在氟化铯的作用下形成偶氮中间体 139; 二者与 $\alpha$-溴代酮 137 发生 $[2+2+2]$ 环化反应生成 140, 再进一步脱水得到目标产物 141. 该反应的优点在 于无需过渡金属催化剂参与，原料简单易得，反应体系 简单，一步完成两个 $\mathrm{C}-\mathrm{N}$ 键和一个 $\mathrm{C}-\mathrm{C}$ 键的构建; 不 足之处在于反应收率不够理想，且仅当 $\mathrm{R}^{2}$ 基团为芳基 时才能发生反应.

\subsection{7 邻溴苯乙酮和邻氨基苯甲酰胖的串联环化反} 应

2017 年, 范学森小组 ${ }^{[80]}$ 报道了以邻溴苯乙酮和邻 氨基苯甲酰肼为原料在 $\mathrm{I}_{2}$ 的介导下发生的氧化-环化串 联反应, 得到了一系列全新骨架的喹唑啉并噌啉酮骨架 类杂环分子(Eq. 27). 该方法具有原料简单易得, 无需

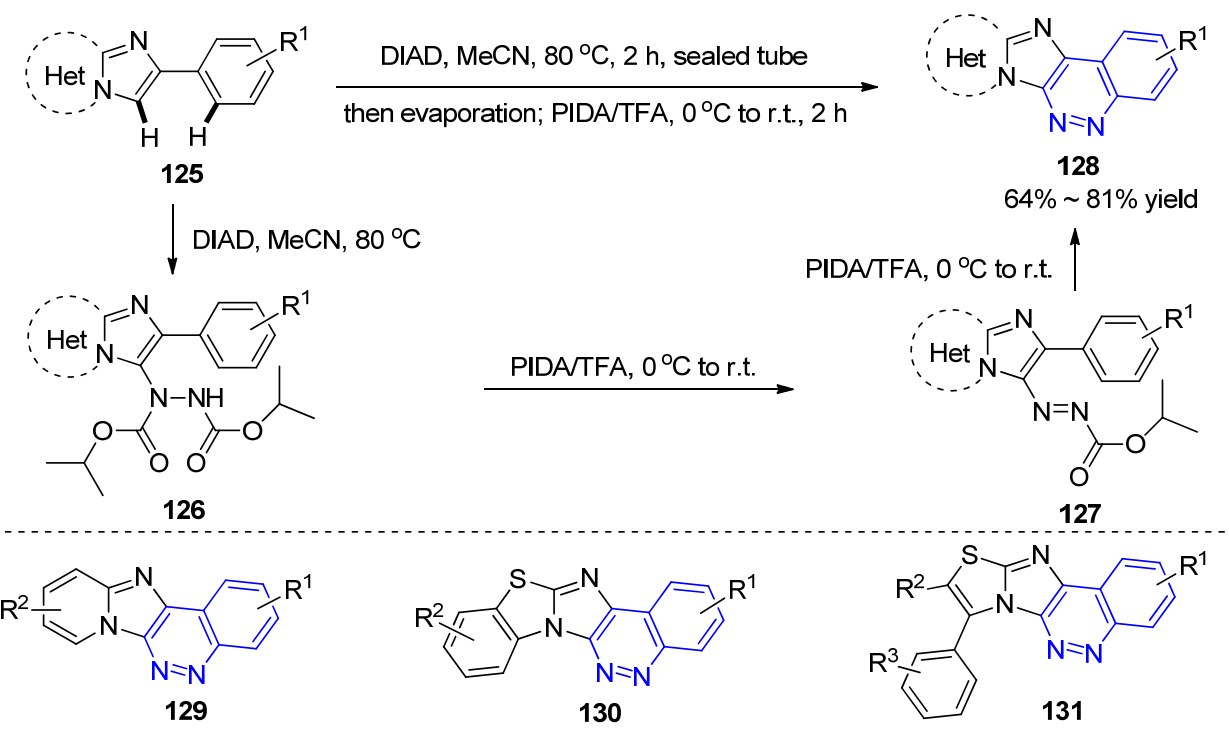

图式 13 C-3 选择性氢胺化/氧化脱氢偶联一锅法合成噌啉化合物

Scheme 13 One-pot synthesis of cinnolines through C-3 regioselective hydrazination and oxidative $N$-arylation 


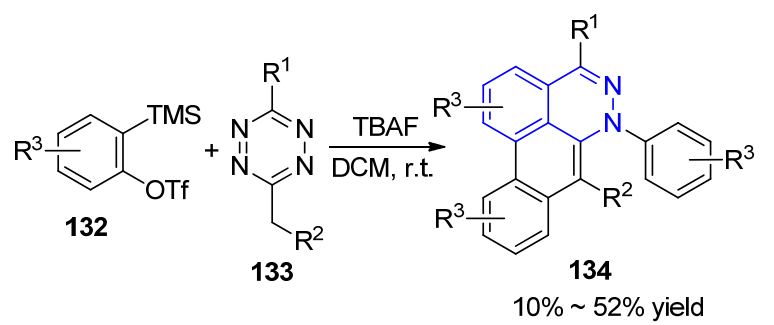

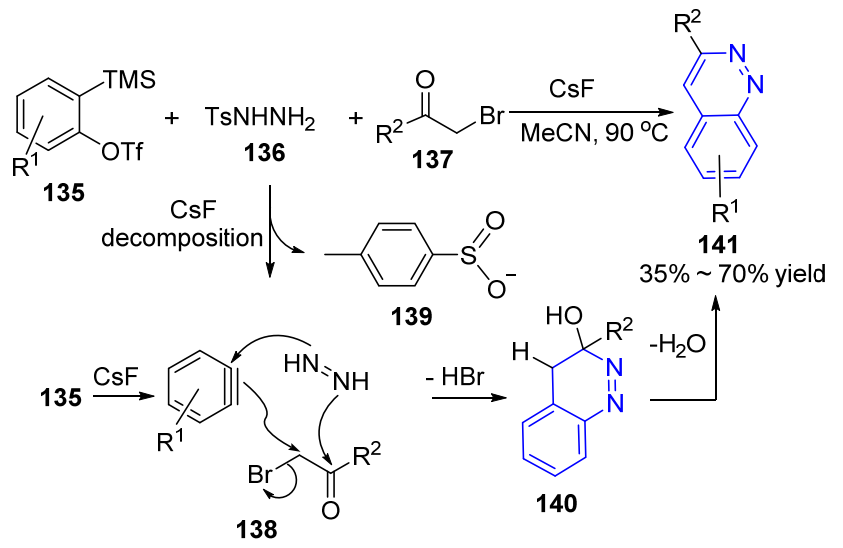

图式 14 苯炔中间体的 $[2+2+2]$ 环化反应 Scheme $14[2+2+2]$ annulation of benzyne

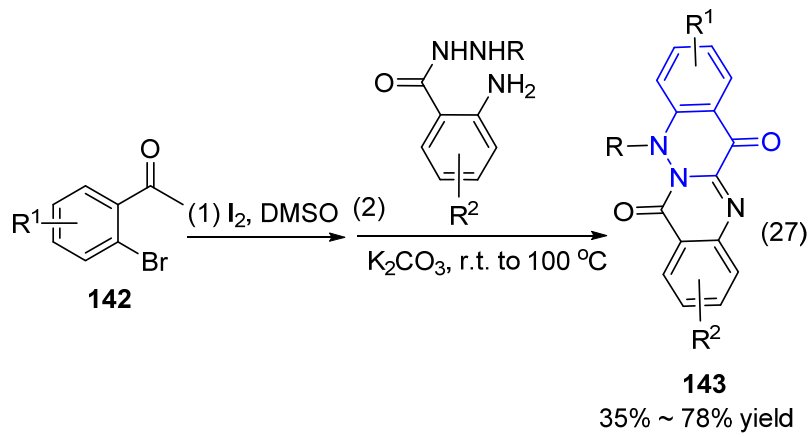

金属催化剂参与，操作简便等优点.

\section{3 总结和展望}

综上所述, 近年来噌啉类化合物的合成新方法呈现 快速发展的趋势, 其中金属催化的偶联反应和 $\mathrm{C}-\mathrm{H}$ 活 化策略等现代合成技术的不断发展为噌啉类化合物的 合成新方法研究注入了强大的活力. 化学家们通过巧妙 的反应设计, 利用定位基和偶联试剂的变化, 建立了许 多合成新方法, 使得噌啉类化合物特别是噌啉并环类化 合物的合成变得直接、高效. 新方法的发展不但为噌啉 类化合物的合成带来了便利, 也大大丰富了其分子多样 性, 因此, 也将促进新型荧光团、有机发光材料和生物 活性分子的发现. 但是, 某些方法也存在催化剂价格昂 贵、区域选择性差以及官能团耐受性差等不利因素, 这
些客观存在的问题是化学家们前进的动力. 相信未来随 着化学家们的不断努力, 噌啉类化合物的合成新方法会 进一步朝着简单、经济、高效、绿色、选择性和耐受性 好的方向发展. 另外，虽然利用许多新方法合成了一系 列全新结构的噌啉类化合物, 但是对其功能性的研究却 往往滞后, 未来多学科的紧密合作将有助于加速噌啉类 化合物的功能性研究发展.

\section{References}

[1] Bekhit, A. A. Boll. Chim. Farm. 2001, 140, 243.

[2] Lewgowd, W.; Stanczak, A. Arch. Pharm. Chem. Life Sci. 2007, 340,65 .

[3] Ruchelman, A. L.; Singh, S. K.; Ray, A.; Wu, X.; Yang, J.-M.; Zhou, N.; Liu, A.; Liu, L.-F.; LaVoie, E. J. Bioorg. Med. Chem. 2004, 12, 795.

[4] Barraja, P.; Diana, P.; Lauria, A.; Passannanti, A.; Almerico, A. M.; Minnei, C.; Longu, S.; Cogiu, D.; Musiu, C.; La Colla, P. Bioorg. Med. Chem. 1999, 7, 1591.

[5] Vaillancourt, V. A.; Larsen, S. D.; Nair, S. K. WO 0170706, 2001 [Chem. Abstr. 2001, 135, 257250]

[6] (a) Siegfried, A.-G. FR 1393596, 1965 [Chem. Abstr. 1965, 63, 2983].

(b) Schatz, F.; Wagner-Jauregg, T. Helv. Chim. Acta 1968, 51, 1919.

[7] Stanczak, A.; Lewgowd, W.; Ochocki, Z.; Pakulska, W.; Szadowska, A. Pharmazie 1997, 52, 91.

[8] Shen, Y.; Shang, Z.; Yang, Y.; Zhu, S.; Qian, X.; Shi, P.; Zheng, J.; Yang, Y. J. Org. Chem. 2015, 80, 5906.

[9] Tsuji, H.; Yokoi, Y.; Sato, Y.; Tanaka, H.; Nakamura, E. Chem. Asian J. 2011, 6, 2005.

[10] Chen, J.-C.; Wu, H.-C.; Chiang, C.-J.; Peng, L.-C.; Chen, T.; Xing, L.; Liu, S.-W. Polymer 2011, 52, 6011.

[11] (a) Leonard, N. J. Chem. Rev. 1945, 37, 269.

(b) Vinogradova, O. V.; Balova, I. A. Chem. Heterocycl. Compd. 2008, 44, 501.

(c) Han, Y.-T.; Jung, J.-W.; Kim, N.-J. Curr. Org. Chem. 2017, 21, 1265 .

(d) Mathew, T.; Papp, A. A.; Paknia, F.; Fustero, S.; Prakash, G. K. S. Chem. Soc. Rev. 2017, 46, 3060.

[12] Richter, V. V. Berichte. 1883, 16, 677.

[13] Senadi, G. C.; Gore, B.; Hu, W.-P.; Wang, J.-J. Org. Lett. 2016, 18, 2890.

[14] (a) Widman, O. Berichte 1884, 17, 722.

(b) Widman, O. Berichte 1909, 42, 4216

(c) Stoermer, R.; Fincke, H. Berichte 1909, 42, 3115.

(d) Stoermer, R.; Gaus, O. Berichte 1912, 45, 3104.

(e) Pang, X.-B.; Zhao, L.-B.; Zhou, D.-G.; He, P.-Y.; An, Z.; Ni, J.-X.; Yan, R.-L. Org. Biomol. Chem. 2017, 15, 6318.

[15] (a) Keneford, J. R.; Simpson, J. C. E. J. Chem. Soc. 1947, 917. (b) Keneford, J. R.; Simpson, J. C. E. J. Chem. Soc. 1948, 354.

(c) Schofield, K.; Theobald, R. S. J. Chem. Soc. 1949, 2404.

[16] (a) Hu, E.; Kunz, R. K.; Rumfelt, S.; Andrews, K. L.; Li, C.; Hitchcock, S. A.; Lindstrom, M.; Treanor, J. Bioorg. Med. Chem. Lett. 2012, 22, 6938.

(b) Yang, H.; Murigi, F. N.; Wang, Z.-J.; Li, J.-F.; Jin, H.-J.; Tu, Z.-D. Bioorg. Med. Chem. Lett. 2015, 25, 919.

(c) Hu, E.; Ma, J.; Biorn, C.; Lester-Zeiner, D.; Cho, R.; Rumfelt, S.; Kunz, R. K.; Nixey, T.; Michelsen, K.; Miller, S. J. Med. Chem. 2012, 55, 4776.

[17] Hennequin, L. F.; Thomas, A. P.; Johnstone, C.; Stokes, E. S. E.; Plé, P. A.; Lohmann, J. J. M.; Ogilvie, D. J.; Dukes, M.; Wedge, S. R.; Curwen, J. O.; Kendrew, J.; Brempt, C. L. J. Med. Chem. 1999, $42,5369$.

[18] (a) Senadi, G. C.; Gore, B. S.; Hu, W. P.; Wang, J. J. Org. Lett. 2016, 18, 2890. 
(b) Khaligh, N. G.; Mihankhah, T.; Johan, M. R.; Ching, J. J. Monatsh. Chem. 2018, 149, 1083.

[19] (a) Neber, P. W.; Knoller, G.; Herrst, K.; Trissler, A. Liebigs Ann. Chem. 1929, 471, 113

(b) Alford, E. J.; Schofield, K. J. Chem. Soc. 1952, 2102.

[20] Shvartsberg, M. S.; Ivanchikova, I. D. Tetrahedron Lett. 2000, 41, 771

[21] Stefańska, B.; Arciemiuk, M.; Bontemps-Gracz, M. M.; Dzieduszycka, M.; Kupiec, A.; Martellib, S.; Borowskia, E. Bioorg. Med. Chem. 2003, 11, 561

[22] (a) Gomaa, M. A. M. Tetrahedron Lett. 2003, 44, 3493.

(b) Ryu, C. K.; Lee, J. Y. Bioorg. Med. Chem. Lett. 2006, 16, 1850.

(c) Lambert, D. J.; Parikh, N.; Messham, S. J.; Edwards, G.; Truong, H. V.; Dempster, N. M.; Drew, M. G. B.; Nahar, L.; Sarker, S. D.; Ismail, F. M. D. Tetrahedron Lett. 2015, 56, 6980.

[23] (a) Barber, H. J.; Washbourn, K.; Wragg, W. R.; Lunt, E. J. Chem. Soc. 1961, 2828.

(b) Barber, H. J.; Lunt, E. J. Chem. Soc., Perkin Trans. 1 1968, 9 , 1156 .

[24] Al-Awadi, N. A.; Elnagdi, M. H.; Ibrahim, Y. A.; Kaul, K.; Kumar, A. Tetrahedron 2001, 57, 1609.

[25] Liu, X.-F.; Chang, H.-F.; Schmiesing, R. J.; Wesolowski, S. S.; Knappenberger, K. S.; Arriza, J. L.; Chapdelaine, M. J. Bioorg. Med. Chem. 2010, 18, 8374.

[26] Awad, E. D.; El-Abadelah, M. M.; Matar, S.; Zihlif, M. A.; Naffa, R. G.; AlMomani, E. Q. Molecules 2012, 17, 227.

[27] Alhambra, C.; Becker, C.; Blake, T.; Chang, A. H.; Damewood, J. R.; Daniels, T.; Dembofsky, B. T.; Gurley, D. A.; Hall, J. E.; Herzog, K. J.; Horchler, C. L.; Ohnmacht, C. J.; Schmiesing, R. J.; Dudley, A.; Ribadeneira, M. D.; Knappenberger, K. S.; Maciag, C.; Stein, M. M.; Chopra, M.; Liu, X.-F.; Christian, E. P.; Arriza, J. L.; Chapdelaine, M. J. Bioorg. Med. Chem. 2011, 19, 2927.

[28] Vinogradova, O. V.; Balova, I. A.; Popik, V. V. J. Org. Chem. 2011, 76, 6937.

[29] Bräse, S.; Dahmen, S.; Heuts, J. Tetrahedron Lett. 1999, 40, 6201.

[30] (a) Kimball, D. B.; Hayes, A. G.; Haley, M. M. Org. Lett. 2000, 2 , 3825 .

(b) Kimball, D. B.; Weakly, T. J. R.; Herges, R.; Haley, M. M. J. Am. Chem. Soc. 2002, 124, 1572.

(c) Kimball, D. B.; Weakly, T. J. R.; Herges, R.; Hayes, A. G.; Haley, M. M. J. Am. Chem. Soc. 2002, 124, 13463.

(d) Kimball, D. B.; Weakly, T. J. R.; Haley, M. M. J. Org. Chem. 2002, 67, 6395

(e) Kimball, D. B.; Haley, M. M. Angew. Chem., Int. Ed. 2002, 41, 3338 .

(f) Young, B. S.; Marshall, J. L.; MacDonald, E.; Vonnegut, C. L.; Haley, M. M. Chem. Commun. 2012, 48, 5166.

(g) Young, B. S.; Köhler, F.; Herges, R.; Haley, M. M. J. Org. Chem. 2011, 76, 8483 .

[31] (a) Ross, S. D.; Kuntz, I. J. Am. Chem. Soc. 1952, 74, 1297. (b) Corbett, J. F.; Holt, P. F. J. Chem. Soc. 1960, 3646.

[32] Hou, Z.; Fujiwara, Y.; Taniguchi, H. J. Org. Chem. 1988, 53, 311.

[33] Dehghanpour, S.; Afshariazar, F.; Assoud, J. Polyhedron 2012, 35, 69.

[34] (a) Braithwaite, R. S. W.; Holt, P. F.; Hughes, A. N. J. Chem. Soc. 1958, 4073 .

(b) Barton, J. W.; Rowe, D. J. Tetrahedron Lett. 1983, 24, 299.

(c) Barton, J. W.; Sheperd, M. K. Tetrahedron Lett. 1984, 25, 4967.

(d) Benin, V.; Kaszynski, P. J. Org. Chem. 2000, 65, 6388.

[35] (a) Bjorsvik, H.-R.; Gonzalez, R. R.; Liguori, L. J. Org. Chem. 2004, 69, 7720.

(b) Elumalai, V.; Bjorsvik, H.-R. ChemistrySelect 2018, 3, 2092.

[36] Corbett, J. F.; Holt, P. F. J. Chem. Soc. 1961, 3695.

[37] Caronna, T.; Fontana, F.; Mele, A.; Sora, I. N.; Panzeri, W.; Viganò, L. Synthesis 2008, 413.

[38] Takeda, Y.; Okazaki, M.; Maruoka, Y.; Minakata, S. Beilstein J. Org. Chem. 2015, 11, 9.

[39] Lee, D. S.; Chatterjee, T; Ban, J.; Rhee, H.; Cho, E. J. Chemis-
trySelect 2018, 3, 2092.

[40] (a) Holt, P. F.; Went, C. W. J. Chem. Soc. 1963, 4099. (b) Al-Mousawi, S. M.; El-Apasery, M. A. Molecules 2012, 17, 6547.

(c) Al-Awadi, N. A.; Ibrahim, Y. A.; Elnagdi, M. H.; Adam, A. Y.; John, E. J. Anal. Appl. Pyrol. 2017, 124, 602.

[41] Wimmer, R.; Müller, N. Monatsh. Chem. 1998, 129, 1161.

[42] Sharma, M.; Maheshwari, A.; Bindal, N. J. Heterocycl. Chem. 2013, 50, 116 .

[43] Swenton, J. S.; Ikeler, T, J.; Williams, B. H. J. Am. Chem. Soc. 1970, 92, 3103.

[44] Yu, Y.; Singh, S. K.; Liu, A.; Li, T.-K.; Liu, L. F.; LaVoie, E. J. Bioorg. Med. Chem. 2003, 11, 1475.

[45] (a) Parrino, B.; Carbone, A.; Muscarella, M.; Spanò, V.; Montalbano, A.; Barraja, P.; Salvador, A.; Vedaldi, D.; Cirrincione, G.; Diana, P. J. Med. Chem. 2014, 57, 9495.

(b) Shen, Y.; Zhang, Q.; Qian, X.; Yang, Y. Anal. Chem. 2015, 87, 1274.

[46] Hasegawa, K.; Kimura, N.; Arai, S.; Nishida, A. J. Org. Chem. 2008, 73, 6363.

[47] Zhu, C.; Yamane, M. Tetrahedron 2011, 67, 4933

[48] Ball, C. J.; Gilmore, J.; Willis, M. C. Angew. Chem., Int. Ed. 2012, 51,5718 .

[49] Kumar, A.; Tiwari, D. K.; Sridhard, B.; Likhar, P. R. Org. Biomol. Chem. 2018, 16, 4840.

[50] Zhao, S.; Yu, R.; Chen, W.; Liu, M.; Wu, H. Org. Lett. 2015, 17 , 2828.

[51] Jurberg, I. D.; Gagosz, F. J. Organomet. Chem. 2011, 696, 37.

[52] Gogoi, P.; Gogoi, S. R.; Devi, N.; Barman, P. Synth. Commun. 2014, 44, 1142 .

[53] Zhang, G.; Miao, J.; Zhao, Y.; Ge, H. Angew. Chem., Int. Ed. 2012 , 51,8318 .

[54] Lan, C.; Tian, Z.; Liang, X.; Gao, M.; Liu, W.; An, Y.; Fu, W.; Jiao, G.; Xiao, J.; Xu, B. Adv. Synth. Catal. 2017, 359, 3735.

[55] Fan, Z.; Wu, K.; Xing, L.; Yao, Q.; Zhang, A. Chem. Commun. 2014, 50, 1682.

[56] Reddy, B. V. S.; Reddy, C. R.; Reddy, M. R.; Yarlagadda, S.; Sridhar, B. Org. Lett. 2015, 17, 3730.

[57] Zhao, D.; Wu, Q.; Huang, X.; Song, F.; Lv, T.; You, J. Chem.-Eur. J. 2013, 19, 6239 .

[58] Guimond, N.; Gouliaras, C.; Fagnou, K. J. Am. Chem. Soc. 2010 $132,6908$.

[59] (a) Muralirajan, K. Cheng, C.-H. Chem.-Eur. J. 2013, 19, 6198. (b) Prakash, S.; Muralirajan, K.; Cheng, C.-H. Angew. Chem., Int. Ed. 2016, 55, 1844.

[60] Xing, L.; Fan, Z.; Hou, C.; Yong, G.; Zhang, A. Adv. Synth. Catal. 2014, 356, 972.

[61] Rajkumar, S.; Savarimuthu, S. A.; Kumaran, R. S.; Nagarajad, C. M.; Gandhi, T. Chem. Commun. 2016, 52, 2509.

[62] Mayakrishnan, S.; Arun, Y.; Balachandran, C.; Emi, N.; Muralidharana, D.; Perumal, P. T. Org. Biomol. Chem. 2016, 14, 1958.

[63] (a) Shi, J.; Zhou, J.; Yan, Y.; Jia, J.; Liu, X.; Song, H.; Xu, H. E.; Yi, W. Chem. Commun. 2015, 51, 668.

(b) Lv, H.; Xu, W. L.; Lin, K.; Shi, J.; Yi, W. Eur. J. Org. Chem. 2016, 5637.

[64] Son, J.-Y.; Kim, S.; Jeon, W. H.; Lee, P. H. Org. Lett. 2015, 46, 2518.

[65] Sharma, S.; Han, S. H.; Han, S.; Ji, W.; Oh, J.; Lee, S.-Y.; Oh, J. S.; Jung, Y. H.; Kim, I. S. Org. Lett. 2015, 46, 2852.

[66] Sun, P.; Wu, Y.; Huang, Y.; Wu, X.; Xu, J.; Yao, H.; Lin, A. Org. Chem. Front. 2016, 3, 91.

[67] Song, C.; Yang, C.; Zhang, F.; Wang, J.; Zhu, J. Org. Lett. 2016, 18 , 4510.

[68] Li, P.; Xu, X.; Chen, J.; Yao, H.; Lin, A. Org. Chem. Front. 2018, 5, 1777.

[69] Chen, X.; Zheng, G.; Song, G.; Li, X. Adv. Synth. Catal. 2018, 360, 2836.

[70] Yang, C.; Song, F.; Chen, J.; Huang. Y. Adv. Synth. Catal. 2017, 
$359,3496$.

[71] (a) Kiselyov, A. S. Tetrahedron Lett. 1995, 36, 1383.

(b) Kiselyov, A. S.; Dominguez, C. Tetrahedron Lett. 1999, 40, 5111.

[72] (a) Kobayashi, S.; Furuya, T.; Otani, T.; Saito, T. Tetrahedron 2008, 64, 9705 .

(b) Süennemann, H. W.; Banwell, M. G.; de Meijere, A. Chem.-Eur. J. 2008, 14, 7236 .

(c) Kobayashi, S.; Furuya, T.; Otani, T.; Saito, T. Tetrahedron Lett. 2008, $49,4513$.

(d) Alajarín, M.; Cabrera, J.; Pastor, A.; Sánchez-Andrada, P.; Bautista, D. J. Org. Chem. 2008, 73, 963.

[73] Alajarin, M.; Bonillo, B.; Marin-Luna, M.; Vidal, A.; Orenes, R.-A.
J. Org. Chem. 2009, 74, 3558.

[74] Chen, D.; Yang, C.; Xie, Y.; Ding, J. Heterocycles 2009, 77, 273.

[75] Li, S.; Zhao, Y.; Wang, K.; Gao, Y.; Han, J.; Cui, B.; Gong, P. Bioorg. Med. Chem. 2013, 21, 2843.

[76] Kandimalla, S. R.; Sabitha, G. RSC Adv. 2016, 6, 67086

[77] Jiao, J.; Xu, L.; Zheng, W.; Xiong, P.; Hu, M.-L.; Tang, R.-Y. Synthesis 2017, 49, 1839.

[78] Suh, S.-E.; Barros, S. A.; Chenoweth, D. M. Chem. Sci. 2015, 6, 5128.

[79] Shu, W.-M.; Ma, J.-R.; Zheng, K.-L.; Wu, A.-X. Org. Lett. 2016 18, 196.

[80] Guo, S.; Zhai, J.; Fan, X. Org. Biomol. Chem. 2017, 15, 1521. 